\title{
Value of flexible bronchoscopy in the pre-operative work-up of solitary pulmonary nodules
}

\author{
Carsten Schwarz*, Nicolas Schönfeld\#, Roland C. Bittner ${ }^{\star}$, Thomas Mairinger ${ }^{+}$, \\ Holger Rüssmann ${ }^{\S}$, Torsten T. Bauer ${ }^{\#}$, Dirk Kaiser ${ }^{\dagger}$ and Robert Loddenkemper ${ }^{\#}$
}

ABSTRACT: The diagnostic value of flexible bronchoscopy in the pre-operative work-up of solitary pulmonary nodules (SPN) is still under debate among pneumologists, radiologists and thoracic surgeons.

In a prospective observational manner, flexible bronchoscopy was routinely performed in 225 patients with SPN of unknown origin.

Of the 225 patients, $\mathbf{8 0 . 5} \%$ had lung cancer, $\mathbf{7 . 6 \%}$ had metastasis of an extrapulmonary primary tumour and $12 \%$ had benign aetiology. Unsuspected endobronchial involvement was found in $4.4 \%$ of all 225 patients (or in $5.5 \%$ of patients with lung cancer). In addition, flexible bronchoscopy clarified the underlying aetiology in $41 \%$ of the cases. The bronchoscopic biopsy results from the SPN were positive in 84 (46.5\%) patients with lung cancer. Surgery was cancelled due to the results of flexible bronchoscopy in four cases (involvement of the right main bronchus (impaired pulmonary function did not allow pneumonectomy) $n=1$, small cell lung cancer $n=1$, bacterial pneumonia $n=2$ ), and the surgical strategy had to be modified to bilobectomy in one patient.

Flexible bronchoscopy changed the planned surgical approach in five cases substantially. These results suggest that routine flexible bronchoscopy should be included in the regular preoperative work-up of patients with SPN.

KEYWORDS: Flexible bronchoscopy, lung cancer, pre-operative evaluation, solitary pulmonary nodule

$\mathbf{T}$ he diagnostic value of flexible bronchoscopy in the pre-operative work-up of solitary pulmonary nodules (SPN) is still under debate among pneumologists, radiologists and thoracic surgeons. There are significant differences in the management of SPN; whereas radiologists tend to recommend short-term follow-up or needle aspiration under computed tomography (CT) guidance, pneumologists and thoracic surgeons prefer a more aggressive approach, especially in patients with a higher likelihood of malignancy [1].

New pulmonary nodules have a high probability of malignancy. In the American College of Chest Physicians (ACCP) evidence-based clinical practice guidelines, studies are cited in which SPN, detected either by screening or incidentally, were malignant in $33-60 \%$ with a diameter measuring $11-20 \mathrm{~mm}$ and in $64-82 \%$ with a diameter measuring $>20 \mathrm{~mm}$ [2]. Smaller pulmonary nodules less often have a malignant aetiology.
The second edition of the ACCP evidence-based clinical practice guidelines recommends a very limited use of bronchoscopy (or transthoracic needle biopsy) in the management of patients who have an indeterminate SPN that measures at least $8-10 \mathrm{~mm}$ in diameter and are candidates for curative (surgical) treatment [3]. The reasoning against routine pre-operative bronchoscopy is that it has been shown to rarely change tumour stage and/or to contraindicate surgery $[4,5]$.

In most German specialised chest hospitals/departments, the endobronchial status is routinely evaluated pre-operatively via flexible bronchoscopy under local anaesthesia in order to: exclude additional endobronchial tumour manifestations; examine vocal cord function; find anatomic variants; and obtain tissue specimens, if possible, from the nodule for pathological analysis, as well as bronchopulmonary secretions for bacteriological examinations [6]. The aim of this strategy is to determine

\section{AFFILIATIONS}

*Charité-Universitätsmedizin Berlin, Christiane Herzog Zentrum, Lungenklinik Heckeshorn, HELIOS Klinikum Emil von Behring, ${ }^{\#}$ Klinik für Pneumologie, Lungenklinik Heckeshorn, HELIOS Klinikum Emil von Behring, "Institut für Diagnostische und Interventionelle Radiologie, Lungenklinik Heckeshorn, HELIOS Klinikum Emil von Behring, +Institut für Pathologie, Lungenklinik Heckeshorn, HELIOS Klinikum Emil von Behring,

${ }^{\S}$ Institut für Mikrobiologie, Immunologie und

Laboratoriumsmedizin, Lungenklinik Heckeshorn, HELIOS Klinikum Emil von Behring, and

${ }^{f}$ Klinik für Thoraxchirurgie,

Lungenklinik Heckeshorn, HELIOS Klinikum Emil von Behring, Berlin, Germany.

\section{CORRESPONDENCE}

R. Loddenkemper

Lungenklinik Heckeshorn

HELIOS Klinikum Emil von Behring Walterhöferstr

10243 Berlin

Germany

E-mail: rloddenkemper@

dgpberlin.de

Received:

Jan 302012

Accepted after revision:

March 222012

First published online:

April 102012 
macroscopic and microscopic findings which might change or obviate the planned surgical approach.

In this prospective study, we wanted to evaluate the potential role of routine pre-operative flexible bronchoscopy in patients with an SPN.

\section{MATERIAL AND METHODS}

The study was conducted at Lungenklinik Heckeshorn (Berlin, Germany) from June 2004 to March 2007 in a prospective observational manner in adults with SPN. We evaluated patients with SPN according to the radiologic definition as a $\leqslant 3 \mathrm{~cm}$ single round opacity, which is well marginated and surrounded by lung parenchyma. There were no associated findings such as atelectasis, hilar enlargement or pleural effusion. Only patients presenting with lesions of unknown origin were included. The nodules had been detected on thoracic CT scans or chest radiographs confirmed by CT. In order to evaluate the tracheobronchial tree and to take biopsies from the SPN for histological or cytological examination, flexible brochoscopy under local anaesthesia was routinely performed in all 225 consecutive patients, after written informed consent was obtained. The study was approved by the Ethics Committee of the Charité-Universitätsmedizin Berlin.

Flexible bronchoscopy and transbronchial biopsy were performed in a bronchoscopy suite by a pneumologist as follows: in the supine position the patient underwent conscious sedation with midazolam, propofol or fentanyl. Local anaesthesia with $2 \%$ lidocaine spray was instilled in the nares and in the throat to provide additional patient comfort. At the beginning of the procedure, a complete inspection of the larynx and the tracheobronchial tree, including the subsegmental bronchi, was performed in order to look for any abnormalities. Next, the SPN was visualised using a C-arm fluoroscope. Biopsies from the nodules were taken under fluoroscopic guidance in $157(77.7 \%)$ out of 225 patients in whom the lesion was reachable. To reach the SPN, a forceps, brush and/or catheter were introduced under C-arm fluoroscopic control. In addition, in all cases bronchial secretions were obtained during flexible bronchoscopy for bacteriological examination. For safety reasons and to exclude pneumothorax, a chest radiograph was taken on the day following forceps biopsies or sooner if clinical symptoms developed.

In patients with indeterminate tissue results from flexible bronchoscopy, surgery was directly planned, but patients with risk factors for surgery were first transferred to the radiologist for CT-guided transthoracic needle biopsy. Depending on the clinical and functional status of the patient, as well as the histological and anatomic findings, either lobectomy, wedge resection, segment resection, atypical resection, bilobectomy or pneumonectomy were performed after exclusion of distal metastases (at that time positron emission tomography (PET) was not yet introduced in our hospital).

\section{Statistical analysis}

Statistical analyses were performed using commercially available software (SPSS Statistics 19; SPSS Inc., Chicago, IL, USA and Microsoft Excel; Microsoft Corp., Redmond, WA, USA). The unpaired t-test and Mann-Whitney tests were used to analyse statistical differences in nodule sizes. A p-value $<0.05$ were regarded as significant. Quantitative variables are presented as median (interquartile range) and categorical variables are presented as proportions.

\section{RESULTS}

In total, 225 patients were included in the study, 135 (60\%) males and $90(40 \%)$ females. The median age was 67 yrs and the mean age was $66 \pm 18$ yrs. Among the 198 patients with malignant aetiology $118(60 \%)$ were male and $80(40 \%)$ were female. Among the 27 patients with benign aetiology, 17 (63\%) were male versus $10(37 \%)$ females. The median age of the patients with malignancies was $67 \mathrm{yrs}$ and the mean age was $67 \pm 9 \mathrm{yrs}$, and in those with benign disease it was 68 yrs and $63 \pm 15$ yrs, respectively.

\section{Radiological results}

The SPNs were located in the right upper lobe in 71 cases, in the left upper lobe in 75 cases, in the right lower lobe in 43 cases, in the left lower lobe in 27 cases and in the middle lobe in nine cases. The size of the SPNs ranged between 0.5 and $3 \mathrm{~cm}$ with a median of $2.5 \mathrm{~cm}$ and a mean of $2.29 \pm 0.6 \mathrm{~cm}$. Mean diameters of malignant and benign nodules were $2.3 \mathrm{~cm}$ and $2.2 \mathrm{~cm}$, respectively (table 1).

\section{Bronchoscopic results}

Flexible bronchoscopy was performed in all 225 patients, usually via the transnasal route. Macroscopically, in 10 cases ( $4.4 \%$ of all cases or $5.5 \%$ of the 181 lung cancer cases) unsuspected malignant involvement of the central bronchial tree was found. Histologically, these proved to be six cases with adenocarcinoma and four with squamous cell cancer. The additional endobronchial tumour manifestations were located in the right upper lobe in three cases (twice segmental bronchus two, lobar bronchus adjacent to the main bronchus $n=1$ ), in the left upper lobe in four cases (twice segmental bronchus one, twice segmental bronchus three), in the left lower lobe bronchus in two cases and in the middle lobe bronchus in one case.

The aetiology of all 225 SPNs is shown in table 2. Biopsies from SPNs were taken in $157(77.7 \%)$ patients during flexible bronchoscopy under fluoroscopic guidance (in the remaining $22.3 \%$ it was not possible to reach the SPN). The histological/ cytological results of these biopsies from SPNs demonstrated malignancy in $88(56 \%)$ cases (histology positive $n=42(48 \%)$, cytology $n=46(52 \%))$, and a benign aetiology in two cases (chondroma $n=1$, granuloma $n=1$ ) (table 2). In addition, two cases of pneumonia were diagnosed bacteriologically. Thus, in total, the underlying morphological (or bacteriological) diagnosis was provided via flexible bronchoscopy in 92 (41\%)

\begin{tabular}{|c|c|c|c|}
\hline \multirow[t]{2}{*}{$\begin{array}{l}\text { Computed tom } \\
\text { pulmonary nod }\end{array}$} & \multirow{2}{*}{\multicolumn{3}{|c|}{$\begin{array}{l}\text { רography size of solitary } \\
\text { dules }\end{array}$}} \\
\hline & & & \\
\hline \multirow{2}{*}{$\begin{array}{l}\text { Mean } \pm S D \mathrm{~cm} \\
\text { Median (interquartile range) } \mathrm{cm}\end{array}$} & $2.29 \pm 0.60$ & $2.3 \pm 0.59$ & $2.2^{\#} \pm 0.63$ \\
\hline & & & $2.5^{\#}(0.5-3.0)$ \\
\hline
\end{tabular}


TABLE 2 Aetiology of 225 cases of solitary pulmonary nodules undergoing pre-operative flexible bronchoscopy and the positive bronchoscopic biopsy results, and the aetiology of 200 cases finally undergoing surgery

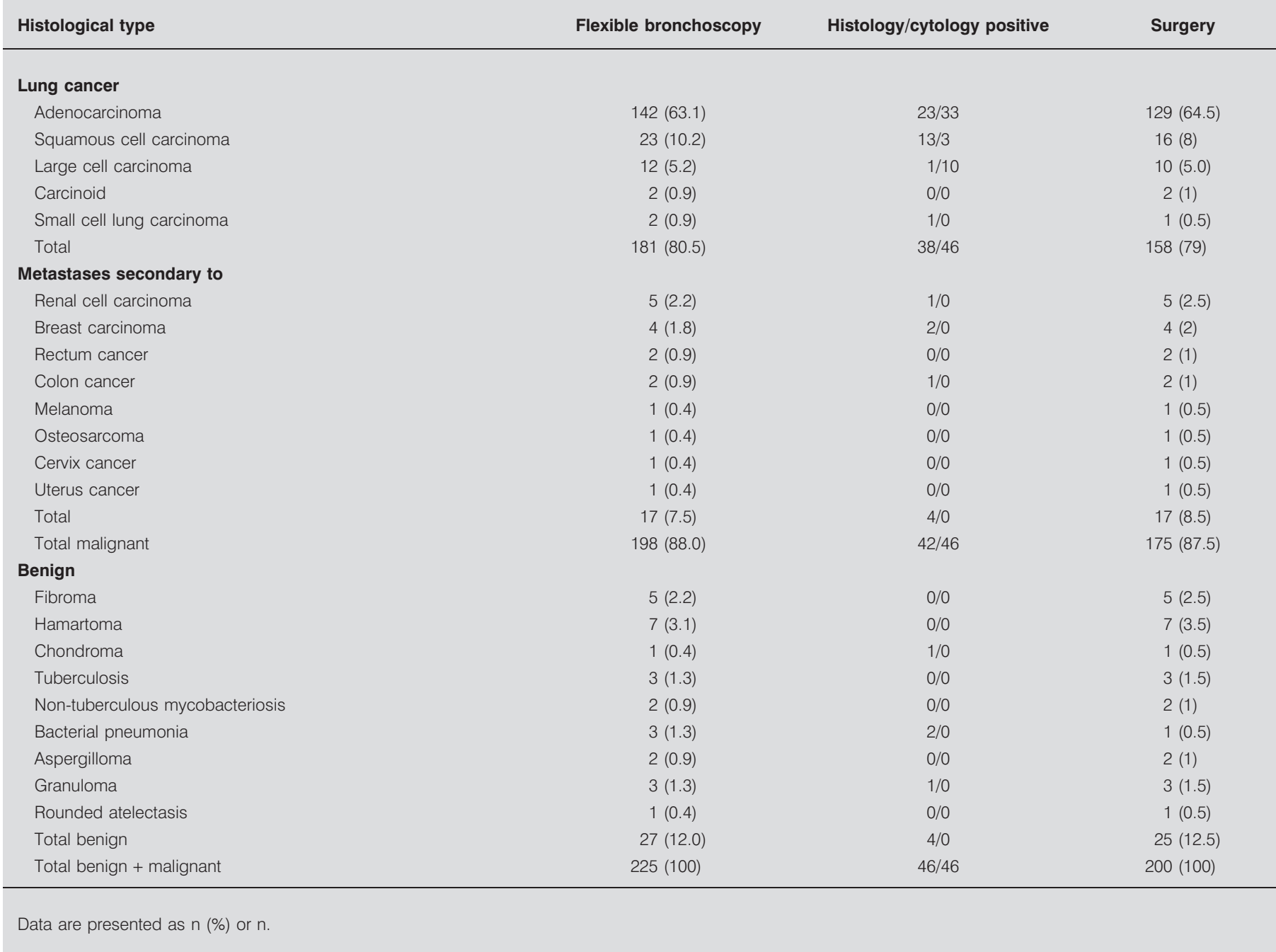

patients (in the 181 lung cancer cases in $46.5 \%$, including one of two small cell lung cancers).

Anatomic variants of smaller degrees (e.g. an accessory segment or two segments instead of three in the right upper lobe) were found in $15(6.7 \%)$ of the 225 patients, but had no impact on the surgical approach.

Flexible bronchoscopy was well tolerated by all patients and there were no serious complications observed, except for one case with a pneumothorax detected radiologically the following day, which resolved spontaneously.

\section{Bacteriological results}

Bacteriological findings in bronchial secretions obtained via flexible bronchoscopy were as follows: oral flora in 205 (91.1\%) cases; Haemophilus influenzae in seven (3.1\%) cases; Streptococcus pneumoniae in two $(0.9 \%)$ cases; Staphylococcus aureus in seven $(3.1 \%)$ cases; Moraxella catarrhalis in two $(0.9 \%)$ cases; and Mycobacterium tuberculosis in two $(0.9 \%)$ cases. In two patients, each with proof of $H$. influenzae and $M$. catarrhalis, in whom the
SPN resolved completely after antibiotic therapy, suggesting pneumonia as the cause, surgery was no longer indicated.

\section{Surgical results}

$200(88.8 \%)$ of the 225 patients underwent surgical resection. Four patients were excluded due to the results of flexible bronchoscopy as mentioned previously, one patient refused surgery and in the other patients the functional risk was considered to be too high due to advanced chronic obstructive pulmonary disease, severe cardiac insufficiency, multimorbidity or age, although primarily a surgical approach was envisaged.

In $175(87.5 \%)$ cases a malignant aetiology was demonstrated histologically, $25(12.5 \%$ ) cases had a benign aetiology (table 2$)$. Lung cancer was the most frequent aetiology with $158(79 \%)$ cases with adenocarcinoma as the leading cell type in $129(81.6 \%)$ cases, followed by squamous cell carcinoma in 16 (10.1\%) cases, large cell carcinoma in $10(6.3 \%)$ cases, carcinoid in two $(1.3 \%)$ cases, and one case of small cell lung cancer (table 2).

The following surgical procedures were used: lobectomy $(n=131)$, segmentectomy $(n=30)$, atypical resection $(n=36)$, 
bilobectomy (one tumour involved middle and lower lobes; $\mathrm{n}=2)$, and pneumonectomy $(\mathrm{n}=1)$. The most frequently applied procedure for lung cancer was lobectomy with systematic mediastinal lymph node dissection (table 3).

\section{Pathological results}

In all 225 cases histological and/or cytological (and/or bacteriological) results were obtained; 17 cases were negative on flexible bronchoscopy and had no operation. In these cases a CT-guided needle biopsy gave the diagnosis (table 2). Nonsmall cell lung cancer was the leading diagnosis with $177(79 \%)$ cases out of 225. Metastatic cancer from different primaries was found in 17 (7.5\%) cases, of which four $(23.5 \%)$ were histologically positive in the bronchoscopic biopsies (table 2).

\section{DISCUSSION}

Routine flexible brochoscopy performed prospectively in the pre-operative work-up of our 225 patients with an SPN revealed unsuspected endobronchial involvement in $4.4 \%$ of the cases (5.5\% of the 181 lung cancer patients). Furthermore, 15 (6.7\%) cases showed an anatomical variant of the tracheobronchial tree. The underlying morphology could be clarified in a considerable number of cases $(41 \%)$ by peripheral bronchoscopic biopsies under fluoroscopic guidance (46.5\% in lung cancer cases). This yield may even be enhanced by additional biopsy techniques, such as needle aspiration with or without endobronchial ultrasound [7-12], but were not included in our routine programme in an otherwise operable SPN. Bacterial pneumonia was found to be the cause of the SPN in two cases.

To our knowledge this is the first prospective study evaluating the role of flexible bronchoscopy in the pre-operative work-up of SPN. In 1993, TORRINGTON and KERN [4] published a retrospective study on the utility of flexible bronchoscopy in the evaluation of SPNs (at that time defined as $\leqslant 6 \mathrm{~cm}$ ). In their 91 cases, flexible bronchoscopy revealed one unsuspected vocal cord carcinoma and five cases with additional submucosal or endobronchial tumour manifestations, which were all in the same anatomic locations and did not affect the planned operative procedure. Since their diagnostic yield of biopsies from SPNs was low (nine out of 30 transbronchial biopsies and only four out of 66 cytological specimens were positive in the cancer patients), they concluded that routine pre-operative flexible bronchoscopy did not measurably benefit patients with SPNs. These results are apparently the reason why routine preoperative flexible brochoscopy should not be recommended in SPN [3]. However, TORRINGTON and KERN [4] stated "that before

\section{TABLE 3 Surgical procedures}

\begin{tabular}{lc} 
Surgical procedure & $\mathbf{n}(\%)$ \\
\hline Lobectomy & $131(65.5)$ \\
Segmentectomy & $30(15)$ \\
Atypical resection & $36(18)$ \\
Bilobectomy & $2(1)$ \\
Pneumonectomy & $1(0.5)$ \\
Total & $200(100)$ \\
\hline
\end{tabular}

abandoning use of this established practice at our institution, we plan to study its yield prospectively". GOLDBERG et al. [5] also came to the conclusion that no additional useful information was derived from flexible bronchoscopy, after retrospectively studying the role of flexible bronchoscopy as a pre-operative staging procedure in only 33 patients.

However, GASPARINI et al. [7] retrospectively examined 570 patients with SPNs (or mass lesions) and detected in 72 (12.6 $\%$ ) visible lesions in the tracheobronchial tree which led to contraindicating surgery in 12 patients and modification of the surgical strategy in 23 patients. ARISTIZABAL et al. [13] retrospectively studied the value of flexible bronchoscopy in addition to chest CT in 64 lung cancer patients with a pulmonary nodule or mass, and detected unsuspected endobronchial involvement in $11(17 \%)$ cases by CT, of which three were true SPNs.

CHHAJED et al. [14] retrospectively assessed the role of routine flexible brochoscopy (combined with PET) in noncalcified pulmonary nodules $\leqslant 3 \mathrm{~cm}$ in size. They observed additional endobronchial tumour manifestations on flexible bronchoscopy in six $(8 \%)$ out of 74 cases, but did not describe if this changed the surgical approach. However, they found PET to be valuable in 19 out of 35 patients with nondiagnostic flexible bronchoscopy and recommended to perform PET if flexible bronchoscopy does not provide a diagnosis. In our hospital PET-CT was only introduced in 2007 and was therefore not applied in this study. The role of PET in the pre-operative work-up of patients with SPN is still under debate [2, 3, 15]. In a review of eight large studies, WAHIDI et al. [2] found a sensitivity of PET imaging of $80-100 \%$ for identifying a malignant SPN, whereas the specificity was much lower (40$100 \%$ ) and more variable. This was confirmed by a recent review of four studies which showed a sensitivity of $88-97 \%$ and a specificity of $83-89 \%$ (with an accuracy of $89-93 \%$ ) [16]. In addition to the differentiation between malignant and benign aetiologies, PET improves the pre-operative staging in lung cancer [17, 18] although the prevalence of distant metastatic disease in stage Ia is low [19].

In our study, 225 patients were included prospectively and all fulfilled the inclusion criteria of SPN as described previously. Among the 225 cases, we found an $88 \%$ of malignancy of which $181(91.4 \%)$ cases were lung cancers. This high proportion most probably results from the pre-selection of patients who are admitted to our specialised chest hospital. Similar results were found in a study of patients undergoing surgical resection for known or suspected malignancy with a rate of $73 \%$ for malignant cancer [20]. In contrast, in a review of lung cancer screening studies, the prevalence of malignant SPN was much lower, varying between 1.1 and $13 \%$ [2]. In a multicentre study in France by AlzAHOuRi et al. [21], out of 11,515 CT scans SPNs were incidentally detected in 152 cases of which 30 (26\%) were malignant.

Unsuspected additional malignant involvement of the central bronchial tree was detected by flexible bronchoscopy in 10 $(5.5 \%)$ of our 181 lung cancer patients, which changed the surgical strategy in two patients: in one a bilobectomy became necessary, in the other, with tumour growth in the right main bronchus, the impaired pulmonary function did not allow a 
pneumonectomy. The finding of a variant of the central tracheobronchial tree in $6.7 \%$ had no impact on the surgical strategy in our cases.

In addition, flexible bronchoscopy provided a diagnosis in 92 (41\%) out of our 225 cases. In 157 (77.7\%) cases biopsies could be taken from the SPN under fluoroscopic guidance demonstrating malignancy in 88 (56.1\%) of these cases, by histology in $47.7 \%$ and by cytology in $52.3 \%$. Thus, in most cases these results obviated the need to obtain an intra-operative histological diagnosis (and convinced some of the patients to agree with the proposed surgical approach). A histological diagnosis was also made in two benign lesions, one chondroma and one granuloma, but both were operated in order to ensure the diagnosis. In two patients with purulent bronchial secretions an unsuspected pneumonia was diagnosed on the basis of the bacteriological results obtained by flexible bronchoscopy, both responded to antibiotic therapy, thus, surgery could be avoided. It is well known that pneumonia may manifest as SPN and may be clinically silent without symptoms [22], as demonstrated in two of our patients.

The pathological results in our 181 lung cancer cases showed adenocarcinoma to be the most frequent cell type $(78,5 \%)$ followed by squamous cell carcinoma $(12.7 \%)$, large cell carcinoma $(6.5 \%)$, small cell carcinoma $(1.0 \%)$ and carcinoid $(1.0 \%)$. This distribution of lung cancer cell types is similar to the data compiled by GoulD et al. [3] from the literature. 17 (7.5\%) cases were solitary metastases due to extrapulmonary primary tumours which were not known before or which were considered unlikely as cause of the SPN; bronchoscopic biopsies demonstrated their malignant aetiology in only four (23.5\%) cases.

Among the 27 cases with an SPN of benign aetiology, tumours such as hamartoma $(n=7)$, fibroma $(n=5)$ and chondroma $(n=1)$ were most frequent. 10 cases had an infectious aetiology (tuberculosis $n=3$, non-tuberculous mycobacteriosis $n=2$, aspergilloma $n=2$, pneumonia $n=3$ ), three cases had a granuloma of indeterminate origin and one a rounded atelectasis.

Furthermore, during bronchoscopy we found 15 anatomic variants of the bronchial tree. This might be of interest for the surgical strategy but had no impact in our study. In addition, sometimes it might be helpful to the surgeon to know the exact segment of the SPN in order to localise it during surgery, in particular if an atypical resection is planned. Flexible bronchoscopy combined with fluoroscopic guidance in addition to CT is a suitable technique to assign the nodule to the corresponding segment.

\section{Conclusions}

In summary, among the 225 cases with an SPN, unsuspected endobronchial involvement was found in 10 lung cancer patients (4.4\% of all SPNs or 5.5\% of all 181 lung cancers). In one case these findings precluded surgery due to functional limitations for the then necessary extension of the surgical approach and in a second case the extension of surgery from lobectomy to bilobectomy was necessary. In two further cases, in which a bacterial pneumonia was diagnosed as the cause of SPN, surgery was rendered unnecessary. Furthermore, since peripheral bronchoscopic biopsies clarified the underlying morphology in a considerable number of cases, our findings suggest that routine flexible bronchoscopy, which is a safe technique, should be included in the pre-operative work-up of patients with SPN. Thus, in our opinion, pre-operative flexible bronchoscopy offers several advantages compared to a bronchoscopy performed at the same time as the planned surgery.

\section{STATEMENT OF INTEREST}

None declared.

\section{REFERENCES}

1 Prosch H, Strasser G, Oschatz E, et al. Management of patients with small pulmonary nodules: a survey of radiologists, pulmonologists, and thoracic surgeons. AJR Am J Roentgenol 2006; 187: 143-148.

2 Wahidi MM, Govert JA, Goudar RK, et al. Evidence for the treatment of patients with pulmonary nodules: when is it lung cancer? ACCP evidence-based clinical practice guidelines (2nd edition). Chest 2007; 132: Suppl. 3, 94S-107S.

3 Gould MK, Fletcher J, Iannettoni MD, et al. Evaluation of patients with pulmonary nodules: when is it lung cancer? ACCP evidencebased clinical practice guidelines (2nd edition). Chest 2007; 132: Suppl. 3, 108S-130S.

4 Torrington KC, Kern JD. The utility of fiberoptic bronchoscopy in the evaluation of the solitary pulmonary nodule. Chest 1993; 104: 1021-1024.

5 Goldberg SK, Walkenstein MD, Steinbach A, et al. The role of staging bronchoscopy in the preoperative assessment of a solitary pulmonary nodule. Chest 1993; 104: 94-97.

6 Goeckenjan G, Sitter H, Thomas M, et al. Prevention, diagnosis, therapy, and follow-up of lung cancer. Interdisciplinary guideline of the German Respiratory Society and the German Cancer Society - abridged version. Pneumologie 2011; 65: 39-59.

7 Gasparini S, Ferretti M, Secchi EB, et al. Integration of transbronchial and percutaneous approach in the diagnosis of peripheral pulmonary nodules or masses: experience with 1,027 consecutive cases. Chest 1995; 108: 131-137.

8 Reichenberger F, Weber J, Tamm M, et al. The value of transbronchial needle aspiration in the diagnosis of peripheral pulmonary lesions. Chest 1999; 116: 704-708.

9 Herth FJ, Eberhardt R, Becker HD, et al. Endobronchial ultrasoundguided transbronchial lung biopsy in fluoroscopically invisible solitary pulmonary nodules: a prospective trial. Chest 2006; 129 : 147-1450.

10 Chao TY, Chien MT, Lie CH, et al. Endobronchial ultrasonographyguided transbronchial needle aspiration increases the diagnostic yield of peripheral pulmonary lesions: a randomized trial. Chest 2009; 136: 229-236.

11 Trisolini R, Cancellieri A, Tinelli C, et al. Performance characteristics and predictors of yield from transbronchial needle aspiration in the diagnosis of peripheral pulmonary lesions. Respirology 2011; 16: 1144-1149.

12 Steinfort DP, Khor YH, Manser RL, et al. Radial probe endobronchial ultrasound for the diagnosis of peripheral lung cancer: systematic review and meta-analysis. Eur Respir J 2011; 37: 902-910.

13 Aristizabal JF, Young KR, Nath H. Can chest CT decrease the use of preoperative bronchoscopy in the evaluation of suspected bronchogenic carcinoma? Chest 1998; 113: 1244-1249.

14 Chhajed PN, Bernasconi M, Gambazzi F, et al. Combining bronchoscopy and positron emission tomography for the diagnosis of the small pulmonary nodule $\leqslant 3 \mathrm{~cm}$. Chest 2005; 128 : 3558-3564.

15 Silvestri GA, Gould MK, Margolis ML, et al. Noninvasive staging of non-small cell lung cancer: ACCP evidenced-based clinical practice guidelines (2nd edition). Chest 2007; 132: Suppl. 3, 178S-201S.

16 Visioni A, Kim J. Positron emission tomography for benign and malignant disease. Surg Clin North Am 2011; 91: 249-266. 
17 Fischer BM, Mortensen J, Hansen $\mathrm{H}$, et al. Multimodality approach to mediastinal staging in non-small cell lung cancer. Faults and benefits of PET-CT: a randomised trial. Thorax 2011; 66: 294-300.

18 Maziak DE, Darling GE, Inculet RI, et al. Positron emission tomography in staging early lung cancer: a randomized trial. Ann Intern Med 2009; 151: 221-228.

19 Viney RC, Boyer MJ, King MT, et al. Randomized controlled trial of the role of positron emission tomography in the management of stage I and II non-small-cell lung cancer. J Clin Oncol 2004; 22: 2357-2362.
20 Isbell JM, Deppen S, Putnam JB Jr, et al. Existing general population models inaccurately predict lung cancer risk in patients referred for surgical evaluation. Ann Thorac Surg 2011; 91: 227-233.

21 Alzahouri K, Velten M, Arveux P, et al. Management of SPN in France. Pathways for definitive diagnosis of solitary pulmonary nodule: a multicentre study in 18 French districts. BMC Cancer 2008; 8, 93: 1-9.

22 Wagner AL, Szabunio M, Hazlett KS, et al. Radiologic manifestations of round pneumonia in adults. AJR Am J Roentgenol 1998; 170: 732-736. 